\title{
The demographic, clinical, and medical manifestations of pulmonary thromboembolism development in COVID-19
}

\author{
Somayeh Sadeghi ${ }^{1,2}$, Maryam Nasirian ${ }^{1,3}$, Elaheh Keivany ${ }^{4,5}$, Peiman Nasri ${ }^{6,7}$, Maryam Sadat Mirenayat ${ }^{8}$ \\ ${ }^{1}$ Infectious Diseases and Tropical Medicine Research Center, Isfahan University of Medical Sciences, ${ }^{2}$ Acquired Immunodeficiency \\ Research Center, Al-Zahra Hospital, Isfahan University of Medical Sciences, ${ }^{3}$ Epidemiology and Biostatistics Department, Health \\ School, Isfahan University of Medical Sciences, ${ }^{4}$ Department of Internal Medicine, School of Medicine, Isfahan University of Medical \\ Sciences, ${ }^{5}$ Department of Pulmonology, Isfahan University of Medical Sciences, ${ }^{6}$ Metabolic Liver Disease Research Center, Isfahan \\ University of Medical Sciences, ${ }^{7}$ Child Growth and Development Research Center, Research Institute for Primordial Prevention of \\ Non-Communicable Disease, Isfahan University of Medical Sciences, Isfahan, ${ }^{8}$ Chronic Respiratory Diseases Research Center, \\ National Research Institute of Tuberculosis and Lung Diseases, Shahid Beheshti University of Medical Sciences, Tehran, Iran
}

p-ISSN 2287-979X / e-ISSN 2288-0011 https://doi.org/10.5045/br.2021.2021131 Blood Res 2021;56:293-300.

Received on July 27, 2021

Revised on October 5, 2021

Accepted on November 4, 2021
*This study was supported by Isfahan University of Medical Sciences.

\section{Correspondence to}

Maryam Sadat Mirenayat, M.D.

Chronic Respiratory Diseases Research Center, National Research Institute of Tuberculosis and Lung Diseases, Shahid Beheshti University of Medical Sciences, Tehran 19839-63113, Iran

E-mail: mirenayat_m@yahoo.com

(C) 2021 Korean Society of Hematology

\section{Background}

Since the emergence of coronavirus disease 2019 (COVID-19), various clinical manifestations ranging from asymptomatic to severe, life-threatening courses have been presented. It is well known that COVID-19 patients are at an increased risk of pulmonary thromboembolism (PTE) development; however, the associated demographic, medical, and clinical factors for developing PTE remain unknown. The current study aimed to assess the characteristics of patients with PTE.

\section{Methods}

This case-control study was derived from an ongoing population-based investigation of hospitalized patients with COVID-19 pneumonia. The case group included 99 patients with PTE confirmed by computed tomography pulmonary angiography (CTPA), and the controls $(\mathrm{N}=132)$ were age-matched patients selected from the PTE-suspected patients with a negative CTPA. The demographic, medical, and clinical characteristics of the study population were entered into the study checklist and compared. A logistic regression test was used to determine the factors associated with PTE development.

\section{Results}

Among the 13,099 admitted patients, 690 (5.26\%) were suspected of having PTE according to their clinical manifestations. CTPA was performed for suspected cases, and PTE was confirmed in 132 patients (19.13\%). Logistic regression assessments revealed that male gender (OR, 2.39; 95\% $\mathrm{Cl}, 1.38-4.13)$, decreased oxygen saturation $(\mathrm{OR}, 2.33$; $95 \% \mathrm{Cl}, 1.27-4.26)$, and lower hemoglobin (OR, 0.83, 0.95), and albumin (OR, 0.31; $95 \% \mathrm{Cl}, 0.18-0.53)$ levels were associated with PTE development.

\section{Conclusion}

PTE was confirmed in one-fifth of suspected patients who underwent CTPA imaging. Male sex, decreased oxygen saturation, and lower levels of hemoglobin and albumin were independent predictors of PTE in patients with COVID-19 pneumonia.

Key Words COVID-19, Pulmonary embolism, Computed tomography angiography, Coronavirus

\section{INTRODUCTION}

Since the emergence of coronavirus disease 2019 (COVID$19)$, various clinical manifestations ranging from asympto- matic disease to symptomatic presentations, including interstitial pneumonia, severe acute respiratory distress syndrome (ARDS), multi-organ failure, and death, have been reported [1]. The respiratory pattern of COVID-19 clinical evolution can be explained in three forms: mild symptomatic involve- 
ment of the upper respiratory tract, non-life-threatening pneumonia, and severe pneumonia with ARDS, which initially presents as non-severe symptoms in the first 7-8 days and rapidly progresses until advanced respiratory support is required [2].

Numerous investigations in the literature have described a hypercoagulable state in patients infected with COVID-19, which leads to thromboembolic events, including pulmonary thromboembolism, coronary artery disease, arterial thrombosis, and ischemic strokes [3-5]. In addition, abnormal coagulation-related parameters have been frequently noted and are associated with a poor prognosis $[6,7]$.

The cytopathic reaction is one of the substantial theories regarding COVID-19 infection pathogenesis; however, from another point of view, the virus induces an unrelenting cascade of local cytokine release, inflammatory response, and a potentially detrimental immune reaction [8]. Excessive inflammatory response, in addition to hypoxia, immobilization, and diffuse intravascular coagulation in the setting of COVID-19 infection, contributes to the prothrombotic state [9].

Pulmonary thromboembolism (PTE) is one of the critical thrombotic conditions estimated to occur in $1.1-3.4 \%$ of COVID-19 patients, regardless of hospital admission. However, the data may have been influenced by the severity of the infection and the short-term follow-up of the patients. Additionally, there is an ongoing debate regarding the necessity for prophylactic and therapeutic anticoagulant use for patients with COVID-19. The current study aimed to assess the characteristics of patients with PTE to determine which COVID-19 patients are at an increased risk for PTE development and to provide subsequent anticoagulation therapy.

\section{MATERIALS AND METHODS}

\section{Study population}

This was a cross-sectional study of patients admitted to Amin and Alzahra Hospitals (affiliated with Isfahan University of Medical Sciences) due to COVID-19 pneumonia from May to June 2020. This case-control investigation was conducted on 231 patients suspected of PTE in two groups, including 99 patients with confirmed PTE according to computed tomography pulmonary angiography (CTPA) and 132 patients whose imaging was negative for PTE.

This study adhered to the tenets of the Declaration of Helsinki. The Institutional Ethical Committee at Isfahan University of Medical Sciences approved the study and all related protocols (IR.MUI.MED.REC.1399.692).

This was an observational study derived from an ongoing cohort study of a large population of COVID-19 pneumonia patients. Accordingly, the patients (or their legal guardian if needed) were informed about the protocols, reassured regarding the confidentiality of their personal information, and written informed consent was obtained from all participants before any intervention.

The study population was gathered from suspected PTE patients hospitalized due to COVID-19 pneumonia, which was confirmed by a positive polymerase chain reaction (PCR) test. Patients with pregnancy, immune deficiency, history of coagulopathies, and thromboembolic events within a month before hospitalization regardless of its type (deep vein thrombosis, pulmonary thromboembolism, cerebrovascular accidents, arterial thrombosis, or myocardial infarction), were excluded from the study. The included patients received anti-COVID-19 infection and anticoagulation therapy according to Iran's national guidelines [10].

\section{Diagnosis of PTE}

Patients with clinical manifestations suggestive of PTE (sudden dyspnea, pleuritic chest pain, hemoptysis, sudden hemodynamic instability, sudden general condition exacerbation, sudden loss of consciousness, and inconsistency of hypoxemia with the severity of lung involvement on computed tomography) were evaluated using D-dimer testing. CTPA was performed to confirm PTE in patients with elevated D-dimer levels [11].

\section{Data collection}

Demographic characteristics, including age, sex, smoking, comorbidities [diabetes mellitus (DM), chronic obstructive pulmonary disease (COPD), end-stage renal disease (ESRD), malignancy, cerebrovascular accident (CVA), ischemic heart disease (IHD), history of PTE], current smoking, and medical history (antiplatelet or anticoagulant administration prior to PTE assessments), were entered into the study checklist.

On admission, hemodynamic information [oxygen saturation $\left(\mathrm{O}_{2}\right.$ saturation), pulse rate, systolic and diastolic blood pressure, respiratory rate, and mobility] and laboratory assessments [complete blood count with differential (CBC diff), albumin, ferritin, C-reactive protein (CRP), D-dimer, prothrombin time (PT), partial thromboplastin time (PTT), international normalized ratio (INR), fibrinogen, troponin, and lactate dehydrogenase (LDH)] were recorded in the study checklist. A reference laboratory performed all assessments to minimize potential bias.

$\mathrm{CRP}$ and $\mathrm{LDH}$ levels were measured every other day during hospital admission, while D-dimer levels were measured twice a week. The greatest difference between the on-admission measurements and the highest parameter level was calculated.

Patients with on-admission oxygen saturation below $90 \%$ and a respiratory rate above 30 breaths per minute were determined to have a severe course of COVID-19 pneumonia.

The patients who were given medications including hydroxychloroquine, antibiotics, remdesivir, interferon, favipiravir, corticosteroids, and kaletra prior to PTE diagnosis, were recruited as well.

Anticoagulation therapy administration status prior to PTE assessment was classified as no anticoagulant therapy, prophylactic, intermediate dose, or therapeutic dose. Anticoagulants were initiated before the incidence of thrombosis. Prophylactic doses included 5,000 IU subcutaneous unfractionated heparin (UFH) (three times a day) [for BMI 
Table 1. Demographic, clinical, and laboratory characteristics of the studied population.

\begin{tabular}{|c|c|c|c|}
\hline & No PTE $(\mathrm{N}=132)$ & PTE $(\mathrm{N}=99)$ & $P$ \\
\hline Age (yr), mean (SD) & $58.0(17.9)$ & $59.0(17.6)$ & 0.665 \\
\hline Gender-male, N (\%) & $63(47.7)$ & $68(68.7)$ & $0.001^{\mathrm{a})}$ \\
\hline \multicolumn{4}{|l|}{ Comorbidities, N (\%) } \\
\hline Diabetes mellitus & $22(16.7)$ & $19(19.2)$ & 0.619 \\
\hline Chronic obstructive pulmonary disease & $8(6.1)$ & $2(2.0)$ & 0.135 \\
\hline End-stage renal disease & $0(0)$ & $3(3.0)$ & - \\
\hline Malignancy & $3(3.0)$ & $3(3.0)$ & 1.000 \\
\hline Cerebrovascular accident & $7(5.3)$ & $6(6.1)$ & 0.805 \\
\hline Ischemic heart disease & $15(11.4)$ & $20(20.2)$ & 0.064 \\
\hline Previous history of pulmonary thromboembolism & $1(0.76)$ & $1(1.0)$ & 0.838 \\
\hline Having the least of one comorbidity & $48(36.4)$ & $38(38.4)$ & 0.753 \\
\hline Current smoking, $\mathrm{N}(\%)$ & $10(7.6)$ & $12(12.1)$ & 0.244 \\
\hline \multicolumn{4}{|l|}{ History of medications, N/N (\%) } \\
\hline None & $31 / 103(30.1)$ & 66/94 (70.2) & $<0.0001^{\mathrm{a})}$ \\
\hline Aspirin & 13/102 (12.8) & 20/94 (21.3) & 0.111 \\
\hline Clopidogrel & $0 / 131(0)$ & 1/99 (1.0) & 0.249 \\
\hline Anticoagulant prophylaxis & 2/132 (1.52) & 3/99 (3.0) & 0.434 \\
\hline Therapeutic anticoagulant & $0 / 132(0)$ & $2 / 99(2.0)$ & 0.156 \\
\hline \multicolumn{4}{|l|}{ On admission clinical presentations } \\
\hline Systolic blood pressure, mean (SD) & $127.1(23.6)$ & $124.1(17.8)$ & 0.297 \\
\hline Systolic blood pressure $<90 \mathrm{mmHg}, \mathrm{N}(\%)$ & $3(2.3)$ & $1(1.0)$ & 0.637 \\
\hline Diastolic blood pressure, mean (SD) & $77.8(15.8)$ & $78.8(13.1)$ & 0.624 \\
\hline Diastolic blood pressure $<60 \mathrm{mmHg}, \mathrm{N}(\%)$ & $3(2.3)$ & $1(1.0)$ & 0.637 \\
\hline Pulse rate per minute, mean (SD) & $92.3(18.6)$ & $94.7(18.0)$ & 0.332 \\
\hline Pulse rate $>100$ per minute, $\mathrm{N}(\%)$ & $33(25.0)$ & $33(33.3)$ & 0.165 \\
\hline Respiratory rate per minute-mean (SD) & $24.5(6.4)$ & $24.6(5.7)$ & 0.838 \\
\hline Respiratory rate $>30$ per minute, $\mathrm{N}(\%)$ & $15(11.4)$ & $13(13.1)$ & 0.684 \\
\hline $\mathrm{O}_{2}$ saturation $(\%)$, mean $(\mathrm{SD})$ & $86.1(8.2)$ & $81.8(10.9)$ & $0.001^{\text {a) }}$ \\
\hline $\mathrm{O}_{2}$ saturation $\mathrm{t}<90 \%, \mathrm{~N}(\%)$ & $83(62.9)$ & $79(79.8)$ & $0.005^{\mathrm{a})}$ \\
\hline $\mathrm{O}_{2}$ saturation $90-93 \%, \mathrm{~N}(\%)$ & $25(18.9)$ & $9(9.1)$ & $0.037^{\mathrm{a})}$ \\
\hline $\mathrm{O}_{2}$ saturation $>93 \%, \mathrm{~N}(\%)$ & $24(18.2)$ & $11(11.1)$ & 0.138 \\
\hline \multicolumn{4}{|l|}{ Clinical presentations three days before CT-scan, $\mathrm{N}(\%)$} \\
\hline Relative bed rest & $75(56.8)$ & $34(40.5)$ & $0.019^{\mathrm{a})}$ \\
\hline Complete bed rest & $77(58.3)$ & $63(75.0)$ & $0.012^{\mathrm{a})}$ \\
\hline \multicolumn{4}{|l|}{ On admission laboratory characteristics, mean (SD) } \\
\hline Neutrophil count (per mL) & $6,042(4,100)$ & $7,851(4,016)$ & $0.001^{\mathrm{a})}$ \\
\hline Lymphocyte count (per mL) & $1,239(1,015)$ & $1,048(1,016)$ & 0.161 \\
\hline Platelet $\times 10^{3}($ per $\mathrm{mL})$ & $193.9(95.7)$ & $216.3(94.4)$ & 0.905 \\
\hline Neutrophil-to-lymphocyte ratio & $6.9(6.9)$ & $10.3(8.1)$ & $0.001^{\mathrm{a})}$ \\
\hline International normalized ratio & $1.23(0.56)$ & $1.27(0.43)$ & 0.544 \\
\hline Hemoglobin $(\mathrm{mg} / \mathrm{dL})$ & $13.3(1.9)$ & $12.6(2.3)$ & $0.006^{\mathrm{a})}$ \\
\hline Ferritin $(\mu \mathrm{g} / \mathrm{L})$ & $814.7(582.5)$ & $817.2(554.7)$ & 0.978 \\
\hline Fibrinogen degradation products $(\mu \mathrm{g} / \mathrm{mL})$ & $25.8(8.9)$ & $26.2(5.8)$ & 0.871 \\
\hline Fibrinogen $(\mathrm{mg} / \mathrm{dL})$ & $331.7(111.4)$ & $278.3(106.5)$ & $0.040^{\mathrm{a})}$ \\
\hline Prothrombin time $(\mathrm{s})$ & $13.8(5.5)$ & $14.1(4.4)$ & 0.721 \\
\hline Partial thromboplastin time (s) & $37.2(15.7)$ & $32.4(8.2)$ & $0.010^{\mathrm{a})}$ \\
\hline Albumin $(\mathrm{g} / \mathrm{dL})$ & $3.64(0.63)$ & $3.25(0.52)$ & $<0.0001^{\text {a) }}$ \\
\hline Troponin $(\mathrm{ng} / \mathrm{mL})$ & $68.2(232.8)$ & $230.0(495.1)$ & $0.019^{\mathrm{a})}$ \\
\hline D-dimer $(\mu \mathrm{g} / \mathrm{mL})$ & $2,869(3,285)$ & $4,775(3,641)$ & $0.001^{\text {a) }}$ \\
\hline C-reactive protein $(\mathrm{mg} / \mathrm{L})$ & $67.6(46.3)$ & $85.2(45.4)$ & $0.006^{\mathrm{a})}$ \\
\hline Lactate dehydrogenase (IU/L) & $794.2(385.1)$ & $1,016.6(527.4)$ & $0.001^{\text {a) }}$ \\
\hline \multicolumn{4}{|l|}{ Maximum laboratory characteristics-Median (IQR) } \\
\hline $\mathrm{D}$-dimer $(\mu \mathrm{g} / \mathrm{mL})$ & $1,971(817-4,732)$ & $3,550(2,259-8,191)$ & $<0.0001^{\mathrm{a})}$ \\
\hline C-reactive protein $(\mathrm{mg} / \mathrm{L})$ & $77(54-111)$ & $98(60-125)$ & $0.034^{\mathrm{a})}$ \\
\hline Lactate dehydrogenase (IU/L) & $803(629-1,215)$ & $1,020(683-1,380)$ & $0.017^{\mathrm{a})}$ \\
\hline \multicolumn{4}{|c|}{ Maximum increase compared to admission time, mean (min-max) } \\
\hline D-dimer $(\mu \mathrm{g} / \mathrm{mL})$ & $575.9(0-8,909)$ & $359.7(0-9,363)$ & 0.050 \\
\hline C-reactive protein $(\mathrm{mg} / \mathrm{L})$ & $11.0(0-102)$ & $5.0(0-86)$ & $0.032^{a)}$ \\
\hline Lactate dehydrogenase (IU/L) & $188.6(0-2,242)$ & $114.3(0-2.644)$ & 0.206 \\
\hline
\end{tabular}

${ }^{a)} \mathrm{Chi}^{2}$ /exact test for categorical variable, independent T-test or Wilcoxon rank-sum test for continuous variable were significant if $P$-value $<0.05$. Abbreviation: PTE, pulmonary thromboembolism. 
$>40 \mathrm{~kg} / \mathrm{m}^{2}: 7,500 \mathrm{IU}$ subcutaneous UFH (three times a day)] or $40 \mathrm{mg}$ subcutaneous enoxaparin (once daily) [for BMI $>40 \mathrm{~kg} / \mathrm{m}^{2}$ : $40 \mathrm{mg}$ subcutaneous enoxaparin (twice daily)]. Intermediate doses included 7,500 IU subcutaneous UFH (three times a day) or $60 \mathrm{mg}$ subcutaneous enoxaparin (daily). The therapeutic doses were determined as $80 \mathrm{IU} / \mathrm{kg} \mathrm{UFH}$ bolus infusion, followed by $18 \mathrm{IU} / \mathrm{kg} / \mathrm{h} \mathrm{UFH}$ infusion, or $1 \mathrm{mg} / \mathrm{kg}$ subcutaneous enoxaparin (twice daily). The doses were defined according to national protocols [10]. Anticoagulantrelated adverse effects, including gastrointestinal (GI) bleeding, hemoptysis, and hematuria, were recorded. Other probable side effects, such as easy bruising, petechiae, or purpura, were categorized as "other".

The latter outcomes were ICU admission requirement, discharge/death, and non-invasive ventilation (NIV)/ intubation.

\section{Data analysis}

The data were entered into the Statistical Package for Social Sciences (SPSS, version 22.0, SPSS Inc., Chicago, IL, USA). The descriptive data were presented as mean and standard deviation or median and range for the continuous variables, and frequency and percentages for categorical variables. The chi-square test or Fisher's exact test was used to compare categorical variables between the groups. Continuous variables were compared using the MannWhitney U test. Binary logistic regression analysis was applied to estimate the odds ratio and determine the association between the assessed factors and thrombotic events in the crude and adjusted models for age and sex. Statistical significance was set at $P<0.05$.

Table 2. Hospital-related characteristics of the studied population.

\begin{tabular}{|c|c|c|c|}
\hline & No PTE & PTE & $P$ \\
\hline Prescribed Drugs & $N=131$ & $N=84$ & - \\
\hline Hydroxychloroquine & $61(46.6)$ & $20(23.8)$ & $0.001^{\mathrm{a})}$ \\
\hline Antibiotic & $118(9.1)$ & $74(88.1)$ & 0.647 \\
\hline Remdesivir & $23(17.6)$ & $14(16.7)$ & 0.866 \\
\hline Interferon & $18(13.8)$ & $4(4.8)$ & $0.034^{\mathrm{a})}$ \\
\hline Favipiravir & $2(1.53)$ & $1(1.20)$ & 0.845 \\
\hline Corticosteroid & $87(66.4)$ & $68(81.0)$ & $0.020^{\mathrm{a})}$ \\
\hline Kaletra & $13(9.1)$ & $7(8.3)$ & 0.695 \\
\hline Unknown & $1(0.75)$ & $15(15.1)$ & \\
\hline Anticoagulation prior to PTE diagnosis, N (\%) & $N=130$ & $N=84$ & - \\
\hline None & $34(26.2)$ & $38(45.2)$ & $0.004^{\mathrm{a})}$ \\
\hline Prophylactic doses & $71(54.6)$ & $27(32.1)$ & $0.001^{\mathrm{a})}$ \\
\hline Intermediate doses & $6(4.6)$ & $8(9.5)$ & 0.156 \\
\hline Therapeutic doses & $19(14.6)$ & $11(13.1)$ & 0.754 \\
\hline Unknown & $2(1.5)$ & $15(15.1)$ & \\
\hline Side effects of anticoagulants, N (\%) & $N=132$ & $N=84$ & - \\
\hline Gl-bleeding & $6(4.6)$ & $6 / 84(7.2)$ & 0.389 \\
\hline Hemoptysis & $8(6.1)$ & $5 / 84(6.0)$ & $0.0001^{\mathrm{a})}$ \\
\hline Hematuria & $2(1.5)$ & $3 / 84(3.6)$ & 0.327 \\
\hline Other & $2(1.5)$ & 3/84 (3.6) & 0.327 \\
\hline Missing & $0(0)$ & $15(15.1)$ & \\
\hline Disease severity $^{\mathrm{a})}, \mathrm{N}(\%)$ & $13(9.9)$ & $10(10.1)$ & 0.949 \\
\hline Hospitalization outcome, N (\%) & $N=132$ & $N=99$ & - \\
\hline Intensive care unit admission & $59(44.7)$ & $47(47.5)$ & 0.675 \\
\hline Non-invasive ventilation & $12(9.1)$ & $25(25.3)$ & $0.001^{\mathrm{a})}$ \\
\hline Intubation & 18 (13.6) & $19(19.2)$ & 0.255 \\
\hline Discharge & $124(93.9)$ & $81(81.8)$ & $0.004^{\mathrm{a})}$ \\
\hline Death & $8(6.1)$ & $18(18.2)$ & \\
\hline Interval times-day, median (IQR) & $N=132$ & $N=99$ & - \\
\hline Symptom to admission & $7(4-10)$ & $7(4-14)$ & 0.467 \\
\hline Symptom to computed tomography, scan & $13(7-17)$ & $14(6-20)$ & 0.841 \\
\hline Admission to computed tomography, scan & $2(0-7)$ & $4(3-8)$ & 0.607 \\
\hline Admission to intensive care unit & $2(0-5)$ & $3(1-6)$ & 0.247 \\
\hline Admission to discharged & $9(5-14)$ & $10(7-19)$ & $0.033^{\mathrm{a})}$ \\
\hline Admission to dead & $13(12-21)$ & $8(13.5-30)$ & 0.837 \\
\hline
\end{tabular}

${ }^{a)} \mathrm{Chi}^{2}$ /exact test for categorical variable, independent T-test or Wilcoxon rank-sum test for continuous variable were significant if $P$-value $<0.05$ and severity considered as $\mathrm{O}_{2}$ sat $<90$ and respiratory rate $>30$. Abbreviation: PTE, pulmonary thromboembolism. 


\section{RESULTS}

Among the 13,099 patients admitted to the reference hospitals due to COVID-19 pneumonia, 690 (5.26\%) were suspected of having PTE according to the clinical manifestations. CTPA was performed for suspected cases $(\mathrm{N}=690)$, among which PTE was confirmed in 132 patients (19.13\%). Ninetynine $(75 \%)$ out of the 132 confirmed PTE patients had complete medical records. Eventually, they were randomly matched with 132 suspected PTE patients with negative CTPA reports.

The PTE patients were predominantly male, had poorer on-admission oxygen saturation levels, higher neutrophil-tolymphocyte ratios (NLR), higher troponin, D-dimer, CRP, and LDH levels, and lower hemoglobin, fibrinogen, PTT, and albumin levels $(P<0.05)$. Detailed information is presented in Table 1.

The hospital-related characteristics of the study population are shown in Table 2. According to this table, the two groups were remarkably different in terms of hydroxychloroquine, interferon, and corticosteroid use. The use of anticoagulants before PTE diagnosis, anticoagulant-related adverse effects, hospitalization outcomes, and the period between admission and discharge were the other parameters that were different between the two groups.

According to logistic regression, the requirement for NIV was significantly increased by PTE in both crude (OR, 3.37; 95\% CI, 1.60-7.12) and adjusted models (OR, 3.40; 95\% CI, 1.59-7.25); however, the other hospitalization outcomes were not associated with PTE incidence (Table 3).

Furthermore, corticosteroid administration was significantly associated with PTE development in crude (OR, 2.14; 95\% CI, 1.11-4.13) and an adjusted model for severe COVID-19 $\left(\mathrm{O}_{2}\right.$ saturation $<90$ and respiratory rate $\left.>30\right)(\mathrm{OR}, 2.17$; 95\% CI, 1.12-4.19).

Logistic regression analysis revealed that male sex and decreased $\mathrm{O}_{2}$ saturation, hemoglobin, and albumin levels were independent predictors of PTE development as shown in Table 4.

\section{DISCUSSION}

The present investigation, which is a secondary study derived from an ongoing report of a large population of hospitalized patients with COVID-19 pneumonia, tried to assess the characteristics of patients with PTE due to severe acute respiratory syndrome coronavirus-2 (SARS-CoV-2) infection. We found that male sex and lower oxygen saturation, hemoglobin, and albumin levels were independently associated with the risk of PTE development. Interestingly, corticosteroid administration led to an approximately 2.5 -fold increased risk of PTE.

Respiratory tract infection is a well-known predisposing factor for PTE among hospitalized patients. Previous studies have shown that roughly $23-30 \%$ of COVID-19 patients were positive for PTE based on CTPA $[12,13]$, a statistic that is in line with the present study in which approximately $20 \%$ of the patients who underwent CTPA had PTE.

Searching the literature shows an increasing body of evidence regarding the increased risk of PTE due to COVID-19. This viral infection predisposes a person to venous thromboembolism due to the activation of a systemic inflammatory response, leading to an imbalance between procoagulant and anticoagulant effects [14].

Higher levels of biomarkers related to the hypercoagulable state induced by COVID-19, including fibrinogen, PTT, INR, and D-dimer, have been noted in our study, which is consistent with data from other studies conducted on PTE patients with COVID-19 pneumonia $[4,6]$. Some studies have attempted to present a cut-off for D-dimer levels to distinguish PTE [15], although most of the recent guidelines did not favor routinely assessing this biomarker to diagnose venous thromboembolism [16]. Likewise, we did not detect D-dimer as a standalone predictor of PTE incidence among patients with SARS-CoV-2.

Male sex was accompanied by a 2-fold increased risk of PTE. Most previous studies have highlighted more severe lung involvement among men than among women $[17,18]$. However, gender-based assessments of PTE due to COVID19 are inadequate. In line with our study, Grillet and colleagues demonstrated that the male gender was a significant

Table 3. The effect of PTE on hospitalization outcomes.

\begin{tabular}{|c|c|c|c|c|c|c|c|}
\hline & & \multirow{2}{*}{ ICU admission } & \multirow{2}{*}{ NIV } & \multirow{2}{*}{ Intubation } & \multirow{2}{*}{ Dead } & \multicolumn{2}{|c|}{ Time from admission to } \\
\hline & & & & & & Discharge & Death \\
\hline \multirow[t]{2}{*}{$\begin{array}{c}\text { OR/exp (Beta })^{a)} \\
(95 \% \mathrm{Cl})\end{array}$} & Crude & $\begin{array}{c}1.11 \\
\left(0.66^{-1.88)}\right.\end{array}$ & $\begin{array}{c}3.37 \\
(1.60-7.12)^{b)}\end{array}$ & $\begin{array}{c}1.50 \\
(0.74-3.04)\end{array}$ & $\begin{array}{c}3.44 \\
\left.(1.43-8.20)^{b}\right)\end{array}$ & $\begin{array}{c}9.42 \\
(0.36-245)\end{array}$ & $\begin{array}{c}8.73 \\
(0.002-302)\end{array}$ \\
\hline & Adjusted $^{\text {b) }}$ & $\begin{array}{c}1.09 \\
(0.64-1.86)\end{array}$ & $\begin{array}{c}3.40 \\
(1.59-7.25)^{\mathrm{b})}\end{array}$ & $\begin{array}{c}1.48 \\
(0.73-3.02)\end{array}$ & $\begin{array}{c}3.41 \\
(1.41-8.28)^{b)}\end{array}$ & $\begin{array}{c}4.25 \\
(0.001-271.9)\end{array}$ & $\begin{array}{c}9.86 \\
(0.39-249.3)\end{array}$ \\
\hline
\end{tabular}

a) Binary logistic regression was used to estimate crude and adjusted odds ratio for categorical variables, and linear logistic regression was used to estimate crude and adjusted exponential beta for time to death and discharge. ${ }^{\text {b) }}$ Adjusted for severity, age, have at least one underlying disease. $P$-value $<0.05$.

Abbreviations: $\mathrm{Cl}$, confidence interval; ICU, intensive care unit; NIV, non-invasive ventilation; OR, odds ratio. 
Table 4. Logistic regression analysis of factors associated with PTE.

\begin{tabular}{|c|c|c|}
\hline & \multicolumn{2}{|c|}{ OR $(95 \% \mathrm{CI})^{\mathrm{c})}$} \\
\hline & Crude & Adjusted $^{\text {b) }}$ \\
\hline Age & $1.00(0.98-1.01)$ & - \\
\hline Gender-male & $2.40(1.39-4.14)^{\mathrm{d})}$ & $2.39(1.38-4.13)^{\mathrm{d}}$ \\
\hline \multicolumn{3}{|l|}{ On admission clinical presentations } \\
\hline $\mathrm{O}_{2}$ saturation percentage & $0.95(0.92-0.98)^{\mathrm{d})}$ & - \\
\hline $\mathrm{O}_{2}$ saturation $<93 \%$ & $2.33(1.27-4.26)^{\mathrm{d})}$ & - \\
\hline Hemoglobin & $0.83(0.73-0.95)^{\mathrm{d})}$ & $0.83(0.73-0.95)^{d}$ \\
\hline Fibrinogen & $0.99(0.98-0.99)^{\mathrm{d})}$ & $0.99(0.99-1.00)$ \\
\hline Albumin & $0.32(0.18-0.53)^{d)}$ & $0.31(0.18-0.55)^{d}$ \\
\hline NLR & $1.07(1.02-1.11)^{\mathrm{d})}$ & $1.07(1.02-1.12)$ \\
\hline Troponin & $1.00(1.00-1.00)^{d)}$ & $1.01(1.00-1.01)^{\mathrm{d}}$ \\
\hline D-dimer & $1.00(1.00-1.00)^{\mathrm{d})}$ & $1.00(1.00-1.00)^{d}$ \\
\hline CRP & $1.00(1.00-1.01)^{\mathrm{d})}$ & $1.00(1.00-1.01)^{\mathrm{d}}$ \\
\hline $\mathrm{LDH}$ & $1.00(1.00-1.00)^{d)}$ & $1.00(1.00-1.00)^{\mathrm{d}}$ \\
\hline \multicolumn{3}{|l|}{ Maximum laboratory characteristics } \\
\hline D-dimer & $1.00(1.00-1.00)^{\mathrm{d})}$ & $1.00(1.00-1.00)^{\mathrm{d}}$ \\
\hline CRP & $1.01(0.99-1.01)$ & $1.01(0.99-1.01)$ \\
\hline LDH & $1.00(0.99-1.00)$ & $1.00(0.99-1.00)$ \\
\hline \multicolumn{3}{|c|}{ Maximum increase compared to admission time } \\
\hline D-dimer & $0.99(0.99-1.00)$ & $0.99(0.99-1.00)$ \\
\hline CRP & $0.98(0.96-0.99)^{\mathrm{d})}$ & $0.98(0.96-0.99)^{d}$ \\
\hline LDH & $0.99(0.99-1.00)$ & $0.99(0.99-1.00)$ \\
\hline Disease severity ${ }^{a)}$ & $1.03(0.43-2.5)$ & - \\
\hline At least have one underlying disease & $1.09(0.63-1.86)$ & $1.05(0.61-1.85)$ \\
\hline Hemoptysis & $0.98(0.30-3.10)$ & $1.01(0.30-3.34)$ \\
\hline
\end{tabular}

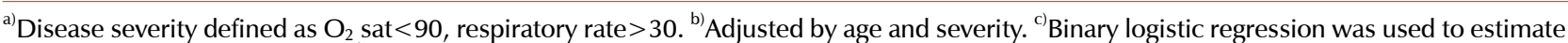
crude and adjusted odds ratio. ${ }^{\text {d) }} P$-value $<0.05$.

Abbreviations: CRP, C reactive protein; LDH, lactate dehydrogenase; NLR, neutrophil lymphocyte ratio.

risk factor for both PTE development and its severity [12]. Nevertheless, a cohort study by Cavagna et al. [19] was opposed. Generally, it is assumed that male sex hormones and the modulatory role of estrogen in immune response intensity and endothelial function are factors associated with a higher risk of complications in males. Similar logic has been noted for other conditions associated with inflammatory processes, such as atherosclerosis. On the other hand, males may consult physicians when in more deteriorated states than females. In addition, males usually have more predisposing factors, such as smoking or other comorbidities [17].

An on-admission oxygen saturation level of less than $93 \%$ was an independent predictor of PTE incidence associated with a 2.33-fold increased risk of PTE. Oxygenation status is a mainstay in the determination of COVID-19 severity, prognosis, and complications. Although PTE itself may lead to a V/Q mismatch, a decreased oxygen saturation level represents a more severe disease course that is accompanied by a storm of cytokine release, oxidative stress, and endothelial dysfunction, and therefore, an increased risk for thromboembolism [1, 20, 21].

Albumin is a well-known marker of health status in medicine. Accordingly, some researchers have tried to assess its value in predicting COVID-19 prognosis. In this regard,
Li et al. [22] presented albumin as an independent predictor of mortality in critically ill patients, and Violi et al. [23] confirmed this theory in a general population of COVID-19 patients that was not limited to critically ill patients. In this study, we have shown up to a $20 \%$ decrease in the risk of PTE by each unit increase in albumin. Nevertheless, no data in this regard, a 4-fold increase in D-dimer levels among patients with hypoalbuminemia reinforces the theory about the association between decreased serum albumin level and hypercoagulable state [16]. Further investigations for the generalization of the data are required.

Hemoglobin was another hematological factor associated with PTE incidence due to COVID-19 pneumonia. Most studies reported insignificant differences between those with thromboembolic events and the control groups [24, 25]. Low levels of hemoglobin negatively affect blood viscosity, which in turn inhibits the function of anti-thrombotic mechanisms due to the reduction of stress formation on endothelial bed dysfunction [26, 27].

Most studies have unanimously described a poor prognosis of PTE in patients with COVID-19 [28, 29]. Surprisingly, we found that PTE independently did not affect any factor related to in-hospital outcomes, including ICU admission, intubation, discharge, and death, except for NIV requirement, which increased up to 3.5 times. Since the emergence 
of COVID-19, NIV has been considered as a key intervention to preserve a patient's appropriate oxygenation and minimize the requirement for invasive strategies. On the other hand, it was assumed that patients under mechanical ventilation were predisposed to PTE development due to their more severe disease course [30]. Thus, the generalization of this outcome requires further investigation.

According to the findings of this study, PTE was confirmed in one-fifth of suspected patients who underwent CTPA imaging. Male sex, decreased oxygen saturation, and lower levels of hemoglobin and albumin were independent predictors of PTE in patients with COVID-19 pneumonia. Further investigations to provide an inexpensive, accessible, non-invasive, and safe scoring system to predict PTE development and initiate anticoagulation therapy for patients with COVID-19 are strongly recommended.

\section{ACKNOWLEDGMENTS}

We are grateful to the officials of the respiratory and infectious diseases wards of Alzahra and Amin Hospitals.

\section{Authors' Disclosures of Potential Conflicts of Interest}

No potential conflicts of interest relevant to this article were reported.

\section{REFERENCES}

1. Guan WJ, Ni ZY, Hu Y, et al. Clinical characteristics of coronavirus disease 2019 in China. N Engl J Med 2020;382:1708-20.

2. De Cobelli F, Palumbo D, Ciceri F, et al. Pulmonary vascular thrombosis in COVID-19 pneumonia. J Cardiothorac Vasc Anesth 2021;35:3631-41.

3. Abou-Ismail MY, Diamond A, Kapoor S, Arafah Y, Nayak L. The hypercoagulable state in COVID-19: incidence, pathophysiology, and management. Thromb Res 2020;194:101-15.

4. Bikdeli B, Madhavan MV, Jimenez D, et al. COVID-19 and thrombotic or thromboembolic disease: implications for prevention, antithrombotic therapy, and follow-up: JACC state-of-the-art review. J Am Coll Cardiol 2020;75:2950-73.

5. Beun R, Kusadasi N, Sikma M, Westerink J, Huisman A. Thromboembolic events and apparent heparin resistance in patients infected with SARS-CoV-2. Int J Lab Hematol 2020; 42(Suppl 1):19-20.

6. Tang N, Li D, Wang X, Sun Z. Abnormal coagulation parameters are associated with poor prognosis in patients with novel coronavirus pneumonia. J Thromb Haemost 2020;18:844-7.

7. Rouhezamin MR, Haseli S. Diagnosing pulmonary thromboembolism in COVID-19: a stepwise clinical and imaging approach. Acad Radiol 2020;27:896-7.

8. Ciceri F, Beretta L, Scandroglio AM, et al. Microvascular COVID-19 lung vessels obstructive thromboinflammatory syndrome (MicroCLOTS): an atypical acute respiratory distress syndrome working hypothesis. Crit Care Resusc 2020;22:95-7.

9. Klok FA, Kruip MJHA, van der Meer NJM, et al. Incidence of thrombotic complications in critically ill ICU patients with COVID-19. Thromb Res 2020;191:145-7.

10. Rahmanzade R, Rahmanzadeh R, Hashemian SM, Tabarsi P. Iran's approach to COVID-19: evolving treatment protocols and ongoing clinical trials. Front Public Health 2020;8:551889.

11. Nagamalesh UM, Prakash VS, Naidu KCK, Sarthak S, Hegde AV, Abhinay T. Acute pulmonary thromboembolism: epidemiology, predictors, and long-term outcome - a single center experience. Indian Heart J 2017;69:160-4.

12. Grillet F, Behr J, Calame P, Aubry S, Delabrousse E. Acute pulmonary embolism associated with COVID-19 pneumonia detected with pulmonary CT angiography. Radiology 2020;296: E186-8.

13. Tirumani SH, Rahnemai-Azar AA, Pierce JD, et al. Are asymptomatic gastrointestinal findings on imaging more common in COVID-19 infection? Study to determine frequency of abdominal findings of COVID-19 infection in patients with and without abdominal symptoms and in patients with chest-only CT scans. Abdom Radiol (NY) 2021;46:2407-14.

14. Lippi G, Favaloro EJ. D-dimer is associated with severity of coronavirus disease 2019: a pooled analysis. Thromb Haemost 2020;120:876-8.

15. Léonard-Lorant I, Delabranche X, Séverac F, et al. Acute pulmonary embolism in patients with COVID-19 at CT angiography and relationship to d-dimer levels. Radiology 2020; 296:E189-91.

16. Thachil J, Tang N, Gando S, et al. ISTH interim guidance on recognition and management of coagulopathy in COVID-19. J Thromb Haemost 2020;18:1023-6.

17. Dangis A, De Brucker N, Heremans A, et al. Impact of gender on extent of lung injury in COVID-19. Clin Radiol 2020;75:554-6.

18. Froldi G, Dorigo P. Endothelial dysfunction in Coronavirus disease 2019 (COVID-19): gender and age influences. Med Hypotheses 2020;144:110015.

19. Cavagna E, Muratore F, Ferrari F. Pulmonary thromboembolism in COVID-19: venous thromboembolism or arterial thrombosis? Radiol Cardiothorac Imaging 2020;2:e200289.

20. Wang $\mathrm{D}, \mathrm{Hu} \mathrm{B}, \mathrm{Hu} \mathrm{C}$, et al. Clinical characteristics of 138 hospitalized patients with 2019 novel coronavirus-infected pneumonia in Wuhan, China. JAMA 2020;323:1061-9.

21. Zhou F, Yu T, Du R, et al. Clinical course and risk factors for mortality of adult inpatients with COVID-19 in Wuhan, China: a retrospective cohort study. Lancet 2020;395:1054-62.

22. Li J, Li M, Zheng S, et al. Plasma albumin levels predict risk for nonsurvivors in critically ill patients with COVID-19. Biomark Med 2020;14:827-37.

23. Violi F, Cangemi R, Romiti GF, et al. Is albumin predictor of mortality in COVID-19? Antioxid Redox Signal 2021;35:139-42.

24. Santoliquido A, Porfidia A, Nesci A, et al. Incidence of deep vein thrombosis among non-ICU patients hospitalized for COVID-19 despite pharmacological thromboprophylaxis. J Thromb Haemost 2020;18:2358-63.

25. Cui S, Chen S, Li X, Liu S, Wang F. Prevalence of venous thromboembolism in patients with severe novel coronavirus pneumonia. J Thromb Haemost 2020;18:1421-4. 
26. Can C, Topacoglu H, Ucku R. Investigation of relationship between blood hemoglobin level and acute pulmonary embolism in emergency setting. Int Med J 2013;20:584-6.

27. Irace C, Scarinci F, Scorcia V, et al. Association among low whole blood viscosity, haematocrit, haemoglobin and diabetic retinopathy in subjects with type 2 diabetes. Br J Ophthalmol 2011;95:94-8.

28. Bellieni A, Intini E, Taddei E, et al. Challenges in COVID-19: is pulmonary thromboembolism related to overall severity? Infect Dis (Lond) 2020;52:585-9.

29. Sakr Y, Giovini M, Leone M, et al. Pulmonary embolism in patients with coronavirus disease-2019 (COVID-19) pneumonia: a narrative review. Ann Intensive Care 2020;10:124.

30. Windisch W, Weber-Carstens S, Kluge S, Rossaint R, Welte T, Karagiannidis $C$. Invasive and non-invasive ventilation in patients with COVID-19. Dtsch Arztebl Int 2020;117:528-33. 